\title{
Varizes do nervo ciático são uma doença pouco conhecida?
}

\author{
Kennedy Gonçalves Pachêco', Fabio Vargas Magalhães², Alexei Loureiro ${ }^{3}$
}

\section{RESUMO}

Objetivo: Relatar nossa experiência com varizes do nervo ciático e indicar que tipo de paciente é mais provável de ser acometido por essa patologia. Método: A amostra foi constituída de 2.400 pacientes, selecionando-se 80 deles (3,3\%) como possíveis portadores de varizes do nervo ciático. Foram considerados pacientes suspeitos: pacientes portadores de varizes na face lateral da perna, coxa e oco poplíteo; pacientes com recidiva anárquica de varizes após safenectomia; pacientes com sintomas e exames positivos para varizes pélvicas; pacientes com história prévia de trombose venosa profunda; pacientes com queixas de dores ciáticas e aumento do diâmetro de um membro com relação ao outro. Esses pacientes foram submetidos a exames detalhados e rigorosos do losango poplíteo com objetivo de identificar varizes dentro da bainha do nervo ciático e na face lateral da perna. Desses, 10 foram submetidos à angiografia venosa para detalhar a relação anatômica com o nervo ciático. Resultados: De 80 pacientes previamente selecionados, encontramos 21 (26,3\%) casos positivos para varizes do nervo ciático ao exame ecográfico. Dez pacientes tinham varizes do nervo ciático associadas a recidiva anárquica de varizes após safenectomia e outros 10 associadas a varizes pélvicas. $A$ dor ciática esteve presente em $100 \%$ dos pacientes positivos para VNC. Conclusão: Varizes do nervo ciático são pouco conhecidas pela medicina. Se o médico fizer uma seleção prévia conforme descrito em "pacientes e métodos" e concentrar-se no exame ecográfico do losango poplíteo e face lateral da perna, irá se surpreender com o elevado número de casos de varizes do nervo ciático que diagnosticará.

\section{PALAVRAS-CHAVE}

Neuropatia ciática, varizes/diagnóstico, nervo isquiático/patologia, síndromes de compressão nervosa.

\section{ABSTRACT}

Nerve varices a little known disease?

Objective: To report our experience with patients with sciatic nerve varices (SNV) and to indicate which type of patient is more likely to be afflicted with this particular pathology. Method: The sample consisted of 2,400 patients, selecting 80 (3.3\%) as possible sufferers of SNV. Patients with the following conditions were highly suspected: patients with varices on the lateral aspect of the leg, the thigh, and oco politeo; patients with uncontrolled variceal recurrence after a safenectomy; patients with symptoms of and who tested positive for pelvic varices; patients with a history of deep vein thrombosis; and patients complaining of sciatic pain along with an increase in the diameter of one limb in relation to the other. These patients underwent detailed and rigorous examination of the "losango popliteo" with the objective of identifying varices inside the sheath of the sciatic nerve and on the lateral aspect of the leg. Of these, 10 underwent venous angiography to detail the anatomical relationship with the sciatic nerve. Results: Of the 80 patients previously selected, we found 21 (26.3\%) cases that were positive for SNV through ultrasound. Ten patients had SNV associated with uncontrolled variceal recurrence after safenectomy, while the other 10 were associated with pelvic varices. Sciatic pain was present in $100 \%$ of the patients who tested positive for SNV. Conclusion: SNV are not well known in Medicine. If the doctor makes a examination as described in the "patients and methods" section and concentrates on the ultrasound scan of the "diamond popliteal" and lateral aspect of the leg, he or she will be surprised by the high number of cases of SNV that will be diagnosed.

\section{KEYWORDS}

Sciatic neuropathy, varicose veins/diagnosis, sciatic nerve/pathology, nerve compression syndromes.

1 Especializado em Flebologia pela L’Université Pierre et Marie Curie, Paris, França, cirurgião vascular e angiologista ex-residente do Hospital de Ipanema, Rio de Janeiro, RJ, Brasil.

2 Ex-residente do Hospital Naval Marcílio Dias, Rio de Janeiro, RJ, Brasil.

3 Ex-residente da Universidade do Estado do Rio de Janeiro (UERJ), Rio de Janeiro, RJ, Brasil. 


\section{Introdução}

As varizes do nervo ciático se caracterizam pela presença de veias varicosas dentro da bainha do nervo ciático, possivelmente causando doença venosa crônica. ${ }^{1}$

O primeiro autor a reconhecer esse tipo de variz foi Verneuil em 1890 apud Van der Stricht. ${ }^{2}$ Em 1988, o jornal francês Phlébologie continha um pequeno artigo escrito por Thiery, ${ }^{3}$ no qual ele escrevia sobre o assunto "varizes do nervo ciático" e afirmava que elas causavam "sintomas varicosos sem varizes" e dores ciáticas começando na região poplítea, irradiando-se para a região glútea.

A apresentação clínica dessa doença poderá se caracterizar como uma dor ciática intermitente na face lateral da perna, irradiando-se para região glútea, acompanhada de varizes originadas na fossa poplítea. ${ }^{4}$

Em 2001, Lemasle et al. ${ }^{5}$ divulgaram 8 casos em $5.000(0,16 \%)$ de varicose da bainha (la gaine) do nervo ciático em pacientes com suspeita de varizes de membros inferiores submetidos a exames.

Em 2001, Labropoulos et al., ${ }^{6}$ num artigo sobre refluxos de veias varicosas de origem não safênica, examinaram 835 pernas com varizes de membros inferiores, encontrando 84 membros com varizes de origem não safênica e nove (1\%) desses apresentavam varizes do nervo ciático.

Lemasle ${ }^{7}$ demonstra que, por meio do ecocolorDoppler venoso, a distribuição dessas varizes pode se exteriorizar já acima da prega da flexão do joelho e mais frequentemente sobre a face posterolateral da panturrilha, por fora da linha mediana. As varizes em questão continuariam pela face lateral da perna, em relação à cabeça do perônio (péroné), dirigindo-se em direção à grande veia safena, caminhando também verticalmente pelo maléolo externo (Figura 1 - Kennedy - e Figura 2 - Lemasle).

Em 2005, Ricci et al. ${ }^{8,9}$ relataram três casos que haviam sido observados em 2003 por ecocolorDoppler venoso, nos quais realizou compressão manual no terço superoposterior de perna, evocando resposta dolorosa ao palpar profundamente essa região.

Bendszus et al. ${ }^{10}$ relataram casos de três pacientes com dor ciática crônica, sem déficit neurológico focal. Eles observaram, por meio de MRI (ressonância nuclear magnética), a presença de varizes glúteas comprimindo o nervo ciático. Os mesmos autores perceberam que

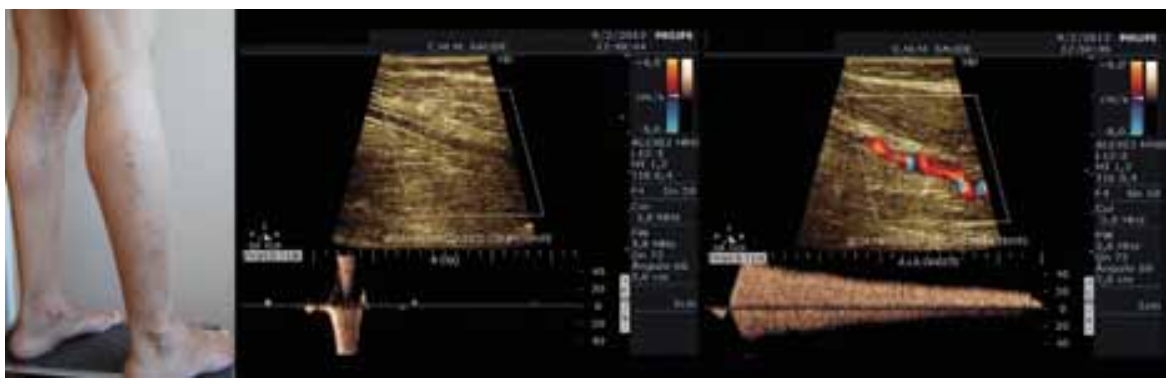

Figura 1 - Kennedy - Varizes do nervo ciático do oco poplíteo à regiáo do nervo fibular.
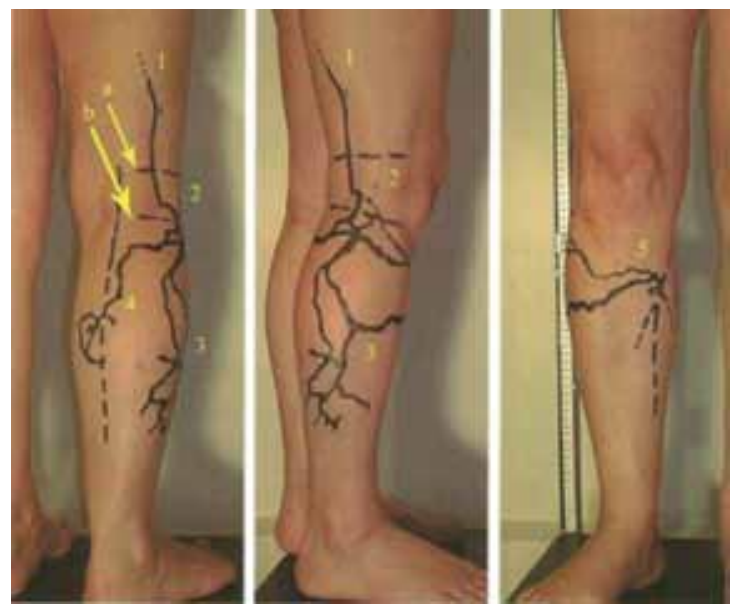

Figura 2 - Lemasle - Distribuição ecoanatômica seguindo orientação do nervo ciático tibial e fibular. 
a descompressão do nervo por cirurgia resultou em completo e permanente alívio das dores.

Gianesini et al., ${ }^{1}$ ao exame de 480 pacientes, encontraram 19 (4\%) casos de varizes do nervo ciático (VNC). Fumagalli e Maltempi ${ }^{11}$ examinaram 92 pacientes, dos quais sete foram positivos para VNC, todos associados a varizes pélvicas.

\section{Materiais e métodos}

Os pacientes estudados pertenciam a etnias e sexo indiferentes, apresentando idade mínima de 27 e máxima de 85 anos, devendo ser portadores de varizes conforme a classificação CEAP.

De um total de 2.400 pacientes atendidos em nossa clínica particular durante um período de seis meses (de agosto de 2011 a janeiro de 2012), selecionamos 80 (3,3\%), os quais consideramos como altamente suspeitos de serem acometidos por varizes do nervo ciático. Essa população classificada era composta por: portadores de varizes localizadas na face lateral de perna, coxa e oco poplíteo; com recidiva anárquica de varizes após safenectomias; sintomatologia e exames positivos para varizes pélvicas; história prévia de trombose venosa profunda; queixas de dor ciática e aumento do diâmetro de um membro em relação ao outro.

Os 80 pacientes selecionados foram submetidos a exame específico de ecocolorDoppler do losango poplíteo, onde se identificou a divisão do nervo ciático próximo à veia e à artéria poplítea. Quando não se identificou o nervo, realizou-se busca ecográfica de varizes dentro dos músculos posterolaterais de coxa, tais como músculo semimembranoso e semitendinoso, posicionando a sonda nos cortes transversos e longitudinais. Para tal, foi utilizado o aparelho de eco da marca Philips com sondas de 7 a $10 \mathrm{MHz}$.

Foram seguidos todos os critérios ecoanatômicos e hemodinâmicos relatados em literatura médica por Lemasle et al. ${ }^{4,5,7}$ Também foi realizada a busca de varizes na face lateral de pernas, investigando a região de nervo fibular, identificando a presença de varizes tubulares e de veias paralelas à veia safena externa (VPS). Quando feita essa identificação, prosseguiu-se com a pesquisa de refluxo por manobras de compressão e descompressão (chasse veineuse) ao nível da panturrilha (compression du Mollet) e dos grupos musculares posterolaterais da coxa.

Sendo assim, consideramos o refluxo como patológico quando o tempo foi acima de 0,5 segundo. Com a finalidade de avaliar a extensão das varizes ou a presença de malformações venosas, realizamos 10 angiotomografias e uma varicografia nos pacientes com diagnóstico positivo para varizes do nervo ciático, por meio do ecocolorDoppler venoso (Figura 3).

Critérios de exclusão: pacientes portadores de neoplasia e/ou insuficiência arterial periférica (índice tornozelo/braço $<0,8)$ e gestantes foram excluídos do estudo.

\section{Metodologia estatística}

Para fins de caracterização, a análise descritiva apresentou sob forma de tabela os dados observados, expressos pela frequência (n) e percentual (\%) para dados categóricos e média, desvio-padrão e mediana para dados numéricos.

Para fins comparativos entre os grupos com e sem VNC, foram aplicados os seguintes métodos: o teste de $\chi^{2}$ ou exato de Fisher para dados categóricos e o teste $t$ de Student para amostras independentes para dados numéricos.

O critério de determinação de significância adotado foi o nível de $5 \%$. A análise estatística foi processada pelo software estatístico SAS System, versão 6.11 (SAS Institute, Inc., Cary, North Carolina).

\section{Resultados}

Durante o período de seis meses (agosto de 2011 a janeiro de 2012) foram examinados 2.400 pacientes, dos quais $80(3,33 \%)$ foram classificados como altamente suspeitos e examinados de forma específica. Observaram-se $21(26,3 \%)$ casos positivos ao exame ecográfico para varizes do nervo ciático. Sendo assim, foi proposto caracterizar e comparar os grupos: VNC positivo $(\mathrm{n}=$ 21) versus $\mathrm{VNC}$ negativo $(\mathrm{n}=59)$, visando evidenciar informações importantes para diagnóstico de VNC.

A tabela 1 demonstra que de 21 pacientes $47,6 \%$ apresentaram recidiva de varizes anárquicas e $47,6 \%$ eram portadores de varizes pélvicas. Além disso, destaca-se a alta frequência de varizes localizadas em face lateral de perna e coxa.

A tabela 1 fornece a frequência (n) e o percentual (\%) das variáveis observadas segundo a VNC (positiva e negativa) e o correspondente nível descritivo ( $p$ valor) do teste estatístico. A análise estatística foi composta pelo teste de $\chi^{2}$ ou exato de Fisher. Os dados numéricos foram expressos pela média \pm desvio-padrão e mediana e comparados pelo teste $t$ de Student para amostras independentes. 

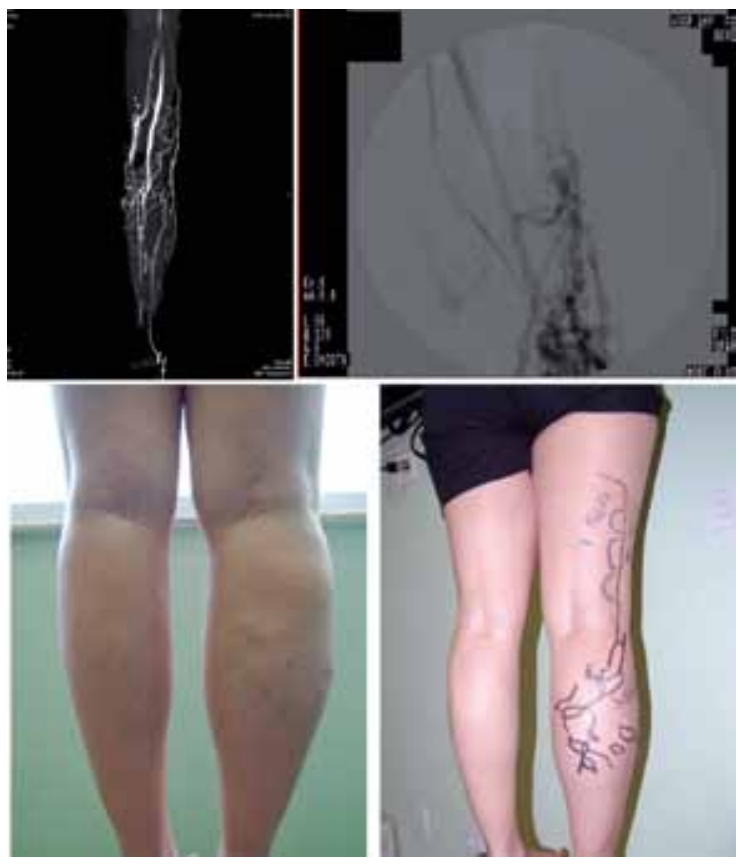

Figura 3 - Angioscanner não seletivo e varicografia com punção da variz do nervo ciático guiado por ecoDoppler.

\begin{tabular}{|c|c|c|c|c|c|c|}
\hline \multirow[t]{2}{*}{ Variável } & \multirow[t]{2}{*}{ Categoria } & \multicolumn{2}{|c|}{ VNC positiva $(\mathrm{n}=21)$} & \multicolumn{2}{|c|}{ VNC negativa $(\mathrm{n}=59)$} & \multirow[t]{2}{*}{$\mathrm{p}$ valor a } \\
\hline & & $\mathrm{n}$ & $\%$ & $\mathrm{n}$ & $\%$ & \\
\hline \multirow[t]{2}{*}{ Sexo } & Masculino & 2 & 9,5 & 11 & 18,6 & \multirow[t]{2}{*}{0,27} \\
\hline & Feminino & 19 & 90,5 & 48 & 81,4 & \\
\hline \multirow[t]{2}{*}{ Recidiva de varizes } & Sim & 10 & 47,6 & 0 & 0,0 & \multirow[t]{2}{*}{$<0,0001$} \\
\hline & Não & 11 & 52,4 & 59 & 100 & \\
\hline \multirow[t]{2}{*}{ História de flebite } & Sim & 3 & 14,3 & 15 & 25,4 & \multirow[t]{2}{*}{0,23} \\
\hline & Não & 18 & 85,7 & 44 & 74,6 & \\
\hline \multirow[t]{4}{*}{ Localização das varizes } & Perna & 10 & 47,6 & 59 & 100 & \multirow[t]{4}{*}{ poucos casos } \\
\hline & Coxa & 1 & 4,8 & 0 & 0,0 & \\
\hline & Perna coxa & 8 & 38,1 & 0 & 0,0 & \\
\hline & Perna posterior & 2 & 9,5 & 0 & 0,0 & \\
\hline \multirow{2}{*}{$\begin{array}{l}\text { Localização das varizes } \\
\text { (agrupada) }\end{array}$} & Só perna & 10 & 47,6 & 59 & 100 & \multirow[t]{2}{*}{$<0,0001$} \\
\hline & Além da perna & 11 & 52,4 & 0 & 0,0 & \\
\hline \multirow[t]{3}{*}{ Lado da perna } & Direita & 8 & 38,1 & 22 & 37,3 & \multirow[t]{3}{*}{0,56} \\
\hline & Esquerda & 7 & 33,3 & 26 & 44,1 & \\
\hline & Ambas & 6 & 28,6 & 11 & 18,6 & \\
\hline \multirow[t]{2}{*}{ Dores ciáticas } & $\operatorname{Sim}$ & 21 & 100 & 59 & 100 & \multirow[t]{2}{*}{ NSA } \\
\hline & Não & 0 & 0,0 & 0 & 0,0 & \\
\hline \multirow[t]{4}{*}{ Aspectos ecocardiográficos } & PAS refluxo & 0 & 0,0 & 59 & 100 & \multirow[t]{4}{*}{$<0,0001$} \\
\hline & Múltiplos canais & 15 & 71,4 & 0 & 0,0 & \\
\hline & Tubular & 2 & 9,5 & 0 & 0,0 & \\
\hline & Tubular+ mult. Canais & 4 & 19,1 & 0 & 0,0 & \\
\hline \multirow[t]{2}{*}{ Varizes pélvicas } & Sim & 10 & 47,6 & 25 & 42,4 & \multirow[t]{2}{*}{0,67} \\
\hline & Não & 11 & 52,4 & 34 & 57,6 & \\
\hline Idade (anos) & Média \pm DP (mediana) & \multicolumn{2}{|c|}{$54,6 \pm 16,1(49)$} & \multicolumn{2}{|c|}{$45,1 \pm 11,7(42)$} & 0,005 \\
\hline CEAP (pontos) & Média \pm DP (mediana) & \multicolumn{2}{|c|}{$3,6 \pm 1,6(3)$} & \multicolumn{2}{|c|}{------- } & NSA \\
\hline
\end{tabular}

a teste de $\chi^{2}$ ou exato de Fisher.

DP: desvio-padrão; NSA: não se aplica. 


\section{Discussão}

Em nosso estudo prospectivo, realizado com amostra seletiva de pacientes, obtivemos dados que nos chamaram a atenção.

De acordo com a metodologia, é aconselhável que se faça uma exploração sistemática com ecocolorDoppler do lado superolateral do losango poplíteo na região em que o nervo ciático se divide nos nervos tibial e fibular e a seguir a face lateral da perna, por onde passa o nervo tibial fibular, e o profissional se surpreenderá com a quantidade de varizes encontradas dentro da bainha do nervo ciático.

Do ponto de vista clínico, nossos casos apresentavam aspectos morfológicos similares aos descritos em literatura médica por Lemasle et al., ${ }^{4,5,7} \mathrm{com}$ presença de varizes na face posterolateral de coxa, oco poplíteo e face lateral de perna, podendo atingir até o maléolo.

Em matéria de exame ecográfico, além da presença de varizes dentro da bainha do nervo ciático, evidenciamos perfurantes de coxa que conectavam o sistema suprafacial com o subfacial, conforme figura 4 .
Destacamos a figura 5, na qual se podem visualizar varizes do nervo ciático atravessando a fáscia oco poplítea, visível tanto por ecoDoppler quanto por angiotomografia seletiva das varizes do nervo ciático, numa paciente com recidiva varicosa depois de várias cirurgias com flebectomias e safenectomias.

A ultrassonografia pode evidenciar uma imagem tubular paralela à safena externa e perfurantes na face lateral da perna, conforme figuras $6 \mathrm{~A} \mathrm{e} \mathrm{B}$, já evidenciado anteriormente por Lemasle, Ricci e Gionesini.

Trigaux et al. ${ }^{12}$ perceberam que nem sempre as varizes do nervo ciático podiam ser visualizadas pelas flebografias tradicionais, e o resultado melhorou quando eles realizaram a varicografia através das varizes que se ligavam às veias do nervo ciático.

Em nossa série, decidimos fazer o mesmo. Puncionamos varizes na face lateral da perna que faziam parte da drenagem das veias do nervo ciático, guiados por aparelho de ecoDoppler, e a seguir injetamos contraste e realizamos uma angiotomografia venosa seletiva, obtendo o resultado conforme a figura 7. Tudo isso, baseado nos estudos prévios de Lemasle et al. ${ }^{5}$ Essa imagem deixou bem clara a relação anatômica das varizes ao lado do nervo ciático até a pelve.

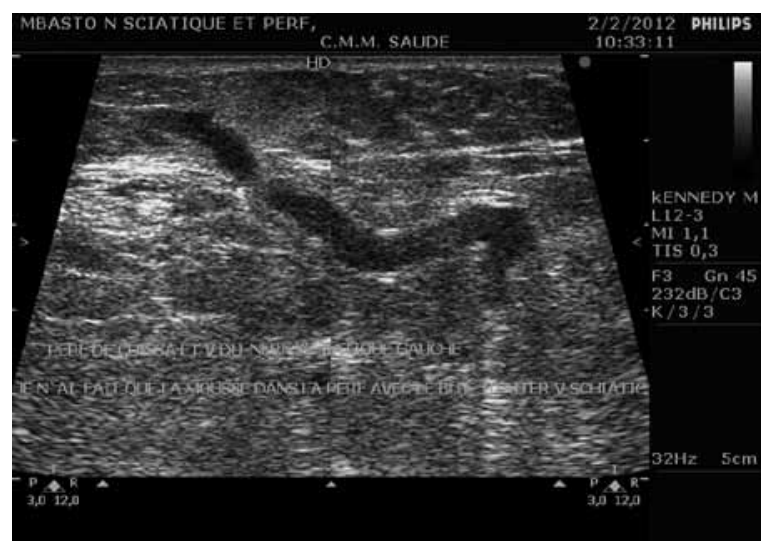

Figura 4 - Perfurante de coxa que liga o sistema suprafacial ao subfacial da variz no nervo ciático.

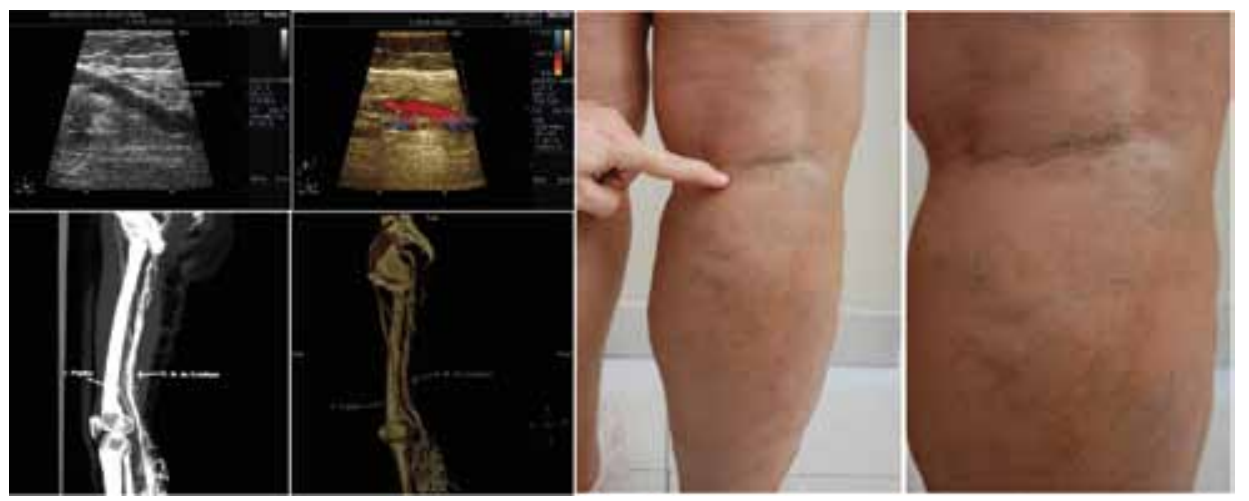

Figura 5 - Recidiva varicosa e passagem das varizes pela fáscia ao nível do oco poplíteo. 


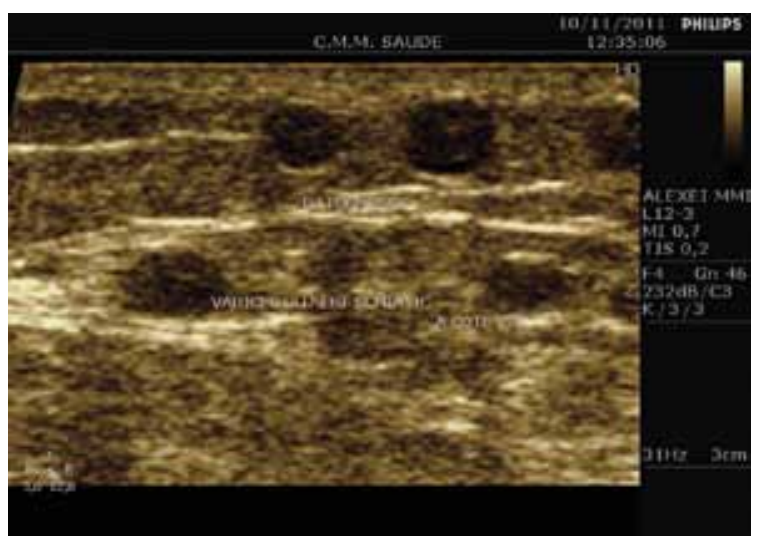

Figura 6A - Segundo Kennedy.

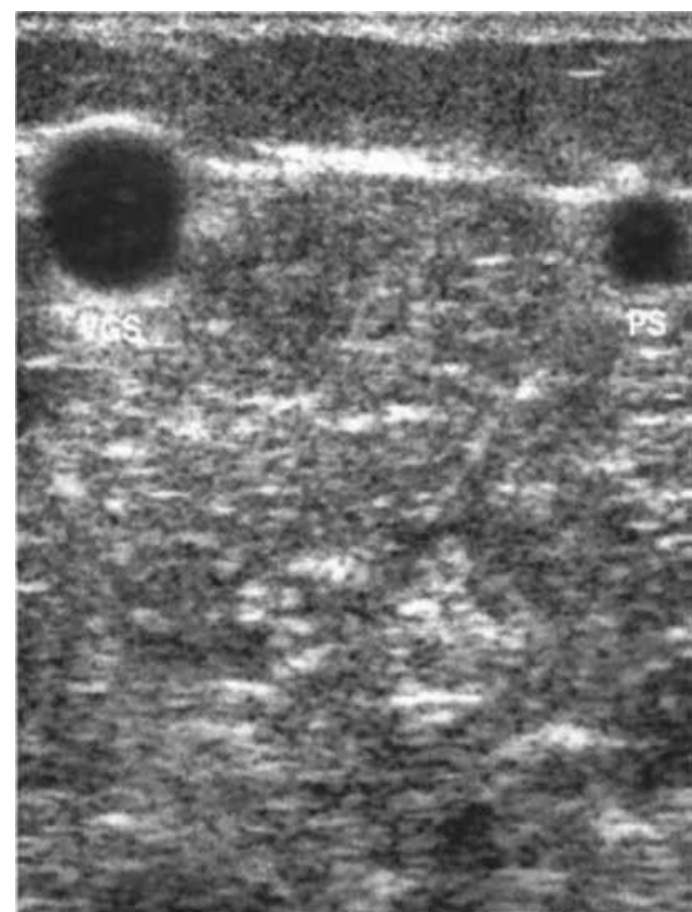

Figura 6B - Segundo Lemasle.

Sob o ponto de vista etiopatogênico, em 21 casos diagnosticados, notamos uma associação importante em 10 casos (Figura 8), com recidiva anárquica de canais safênicos, em outros 10 casos com varizes pélvicas, e em apenas um caso, associação com síndrome pós-trombótica. Trigaux et al. ${ }^{12}$ descrevem sete casos de varizes do nervo ciático, dentre os quais seis apresentavam insuficiência do sistema venoso profundo, e publicaram estudo de recidivas de varizes por meio de flebovaricografia nos quais notaram uma via ciática venosa de drenagem.

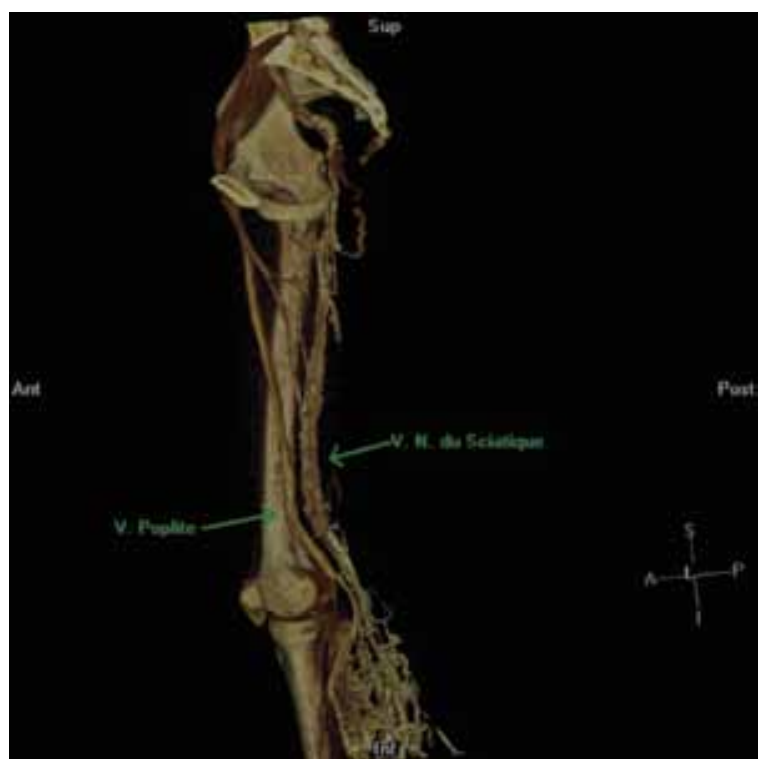

Figura 7 - Angiotomografia seletiva após punção das varizes do nervo ciático na face lateral da perna (região do nervo fibular) guiada por eco.

Van der Stricht ${ }^{2}$ defende a teoria de que, após uma trombose venosa profunda, há utilização das veias do nervo ciático como importantes veias de drenagem.

Creton e Hannequin ${ }^{13}$ e Fumagalli e Maltempi ${ }^{11}$ relatam a relação das varizes do nervo ciático com as varizes pélvicas.

Encontramos pacientes com varizes do nervo ciático de um lado e do outro lado sem varizes do nervo ciático, mas com importante insuficiência da safena interna e da perfurante do pé evoluindo com úlcera no dorso do pé (Figura 9).

Sob o ponto de vista sintomatológico, a dor ciática pode induzir a suspeita de varizes do nervo ciático, porém vale lembrar que causas ortopédicas e neurológicas também podem causar esses sintomas.

Bendszus et al. ${ }^{10}$ descreveram três casos de pacientes que apresentavam dor ciática crônica causada por varizes do nervo ciático, os quais sentiam dor ao sentar ou deitar do lado afetado.

Van Gompel et al. ${ }^{14}$ descreveram quatro pacientes apresentando dores com irradiação para a perna por causa do envolvimento do nervo ciático, todos com etiologia vascular. Esses pacientes foram submetidos a extensa e limitada neurólise do nervo ciático. O estudo histológico no ato cirúrgico confirmou a presença de um angioma venoso, malformação arteriovenosa, malformação venosa associada à síndrome de KlippelTrenaunay e um hemangioma capilar. 


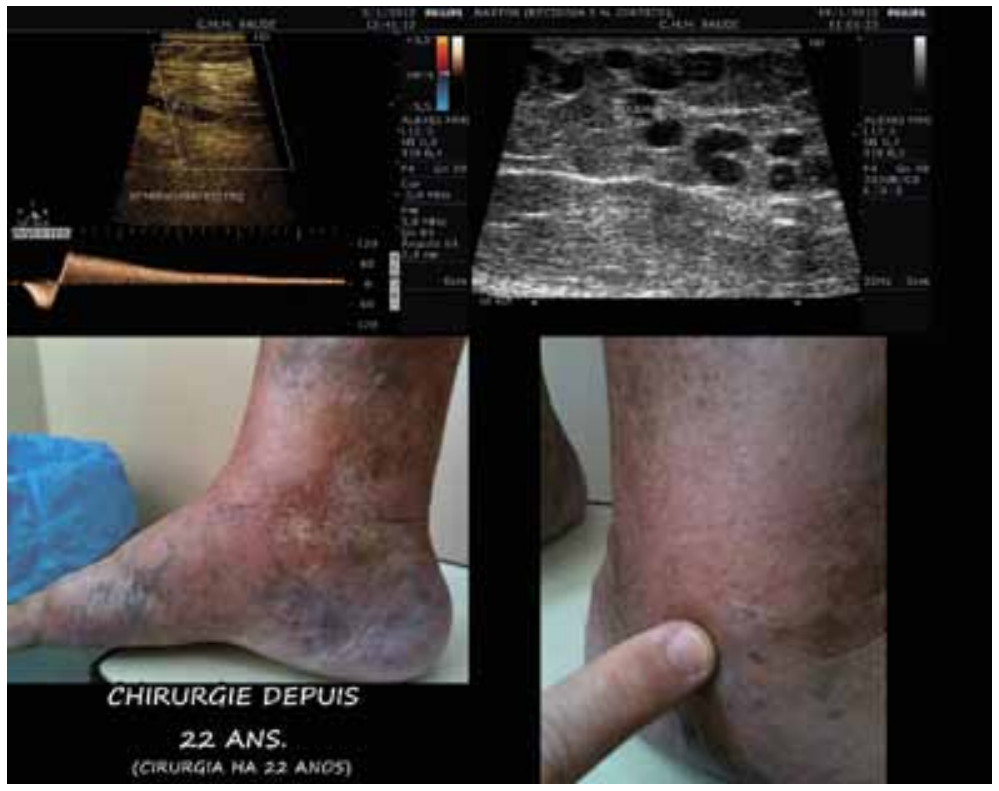

Figura 8 - Demonstra recidiva anárquica e varizes do nervo ciático.
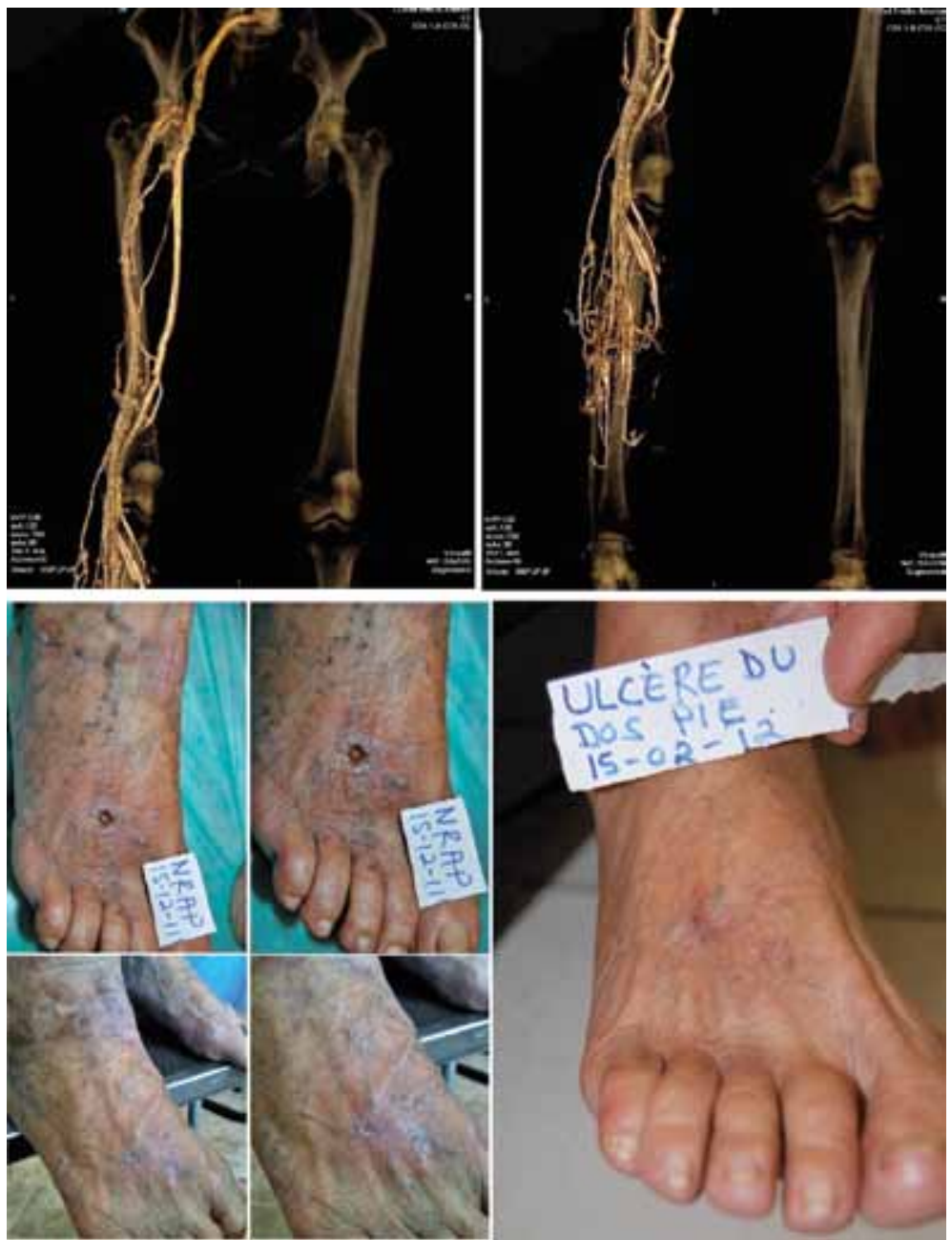

Figura 9 - Úlcera do dorso do pé à direita e varizes do nervo ciático à esquerda tratada com espuma densa guiada por eco. 


\section{Tratamento}

São poucos os artigos em literatura médica mundial que descrevem o tratamento de varizes do nervo ciático, quer seja com cirurgia ou com escleroterapia, assim como o resultado em longo prazo.

Gianesini et al. ${ }^{1}$ relataram bons resultados com escleroterapia em 19 pacientes tratados com espuma densa guiada por eco. Lemasle et al. ${ }^{5}$ trataram com cirurgia e escleroterapia, enquanto Ricci et al. ${ }^{8}$ e Marongiu et al. ${ }^{15}$ destacaram a interrupção cirúrgica, quando possível, das conexões venosas que ligam o sistema venoso posterior acometido pela doença com a veia da bainha do nervo ciático, veias safenas externas, prolongamento axial da veia safena externa (VPS), perfurantes do oco poplíteo e as eventuais ligações com os eixos femorais, obtendo alívio dos sintomas neurológicos.

Leal Monedero et al. ${ }^{16}$ e Creton e Hannequin ${ }^{13}$ encontraram o refluxo venoso originado na veia hipogástrica e realizaram a embolização seletiva com coils e polidocanol, técnica conhecida como "técnica do sandwich", obtendo bons resultados.

Van Gompel et al. ${ }^{14}$ realizaram incisões cirúrgicas extensas na região posterior de coxa e executaram neurólise interna e externa em pacientes com queixa de dor ciática de etiologia vascular. Fumagalli e Maltempi ${ }^{11}$ advertiram que, se for feita a opção pela espuma densa guiada por eco, deve-se evitar fazer na coxa, realizando, sempre que possível, a punção abaixo da prega do joelho, evitando a lesão do nervo ciático ou tibial. Em nossa série, só tratamos 4 de 21 pacientes diagnosticados. Em um deles, foi feita injeção de espuma densa por uma colateral do plano suprafascial que se ligava a uma perfurante, que conduziu o medicamento até as varizes do nervo ciático. As demais punções foram feitas abaixo da região posterior do joelho, e em curto prazo observamos melhora surpreendente das dores dos pacientes. Utilizamos polidocanol na concentração de $0,50 \%$ a $1,5 \%$, na quantidade de 2 a $4 \mathrm{ml}$ em várias sessões.

\section{Conclusão}

As varizes do nervo ciático são uma patologia considerada rara e pouco conhecida na medicina.

Segundo nossa experiência, essa doença apresenta frequência elevada, sendo evidenciada quando se faz uma seleção clínica prévia e criteriosa e se intensifica a investigação diagnóstica por meio de ecocolorDoppler venoso, desde o losango poplíteo, seguindo a orientação do nervo ciático, tibial e fibular.

\section{Referências}

1. Gianesini S, Menegatti E, Tacconi G, Scognamillo F, Liboni A, Zamboni P. Echo-guided foam sclerotherapy treatment of venous malformation involving the sciatic nerve. Phlebology. 2009;24(1):46-7.

2. Van der Stricht J. Varices du nerf sciatique. Phlébologie. 1999;52:349-50.

3. Thiery L. [The vein of the sciatic nerve. Clinical and therapeutic impact]. Phlebologie. 1988;41(3):687-9.

4. Lemasle P. De la clinique aux ultrasons: varicose du nerf sciatique. Phlébologie. 2003;56:67-8.

5. Lemasle P, Uhl JF, Lefebvre-Viladerbo M, Gillot C, Band JM, Vin F. Veine du nerf sciatique et maladie variqueuse. Aspects écho-anatomiques et hemodinamiques. Phlébologie. 2001;54:219-28.

6. Labropoulos N, Tiongson J, Pryor L, Tassiopoulos AK, Kang SS, Mansour MA, et al. Nonsaphenous superficial vein reflux. J Vasc Surg. 2001;34(5):872-7.

7. Lemasle P, Uhl JF. Atlas d'echo-anatomie veineuse superficielle. Paris: Ipsen; 2004.

8. Ricci S, Georgiev M, Jawien A, Zamboni P. Sciatic nerve varices. Eur J Vasc Endovasc Surg. 2005;29(1):83-7.

9. Ricci S. Ultrasound observation of the sciatic nerve and its branches at the popliteal fossa: always visible, never seen. Eur J Vasc Endovasc Surg. 2005;30(6):659-63.

10. Bendszus M, Rieckmann P, Perez J, Koltzenburg M, Reiners $\mathrm{K}$, Solymosi L. Painful vascular compression syndrome of the sciatic nerve caused by gluteal varicosities. Neurology. 2003;61(7):985-7.

11. Fumagalli MD, Maltempi P. La varicose de la gaine du nerf sciatique: faits cliniques. Phlébologie. 2008;61(1):89-93.

12. Trigaux JP, Vanbeers BE, Delchambre FE, De Fays FM, Schoevaerdts JC. Sciatic venous drainage demonstrated by varicography in patients with a patent deep venous system. Cardiovasc Intervent Radiol. 1989;12(2):103-6

13. Creton D, Hannequin L. Insuffisance veineuse pelvienne chez la femme présentant des varices périnéales. Corrélation anatomo-clinique, traitement par embolisations et résultats (31 cas). Phlébologie. 2003;56:257-64.

14. Van Gompel JJ, Griessenauer CJ, Scheithauer BW, Amrami KK, Spinner RJ. Vascular malformations, rare causes of sciatic neuropathy: a case series. Neurosurgery. 2010;67(4):1133-42

15. Marongiu GM, Piredda F, Bacciu PP. The varices of the sciatic nerve: nosologic aspects and our experience. In: Atti 4 Congresso. Collegio It. Flebologia. Ferrara 12-14 Ottobre, 2000. p. 140.

16. Monedero JL, Ezpeleta SZ, Crimberg M, Correa LV, Gutierrez AJ. Subdiaphragmatic venous insufficiency. Embolization treatment using mixed technique (coils and foam). Phlebolymphlogy. 2004;45:269-75.

\footnotetext{
Endereço para correspondência

Kennedy Goncalves Pacheco

Rua Alcindo Guanabara, 25, sala 1304 - Centro (Cinelândia)

20031-130 - Rio de Janeiro, RJ

Telefone: (21) 9811-8965

E-mail: kennedy-gp@uol.com.br
} 